
\title{
Research Sware \\ Stability Analysis for Linear Uncertain Switched Systems in Infinite-time Domain
}

\section{Yadong Shu ( $\nabla 972718523 @ q q . c o m$ )}

Nanjing University of Information Science and Technology https://orcid.org/0000-0001-5887-2264

\section{Bo Li}

Nanjing University of Finance and Economics

\section{Research Article}

Keywords: Uncertain switched systems, Stability in measure, Almost sure stability, Stability in mean, Infinite-time domain

Posted Date: November 29th, 2021

DOl: https://doi.org/10.21203/rs.3.rs-591980/v1

License: (c) (1) This work is licensed under a Creative Commons Attribution 4.0 International License. Read Full License 


\title{
Stability Analysis for Linear Uncertain Switched Systems in Infinite-time Domain
}

\author{
Yadong Shu*, Bo $\mathrm{Li}^{\dagger}$ \\ * School of Mathematics and Statistics, Nanjing University of Information Science and Technology, \\ Nanjing 210044, China \\ $\dagger$ School of Applied Mathematics, Nanjing University of Finance and Economics, Nanjing 210023, China
}

\begin{abstract}
In this work, an uncertain switched system expressed as a series of uncertain differential equations is considered in depth. Stability issues have been widely investigated on switched systems while few results related to stability analysis for uncertain switched systems can be found. Due to such fact, three different stabilities, including stability in measure, almost sure stability and stability in mean, are comprehensively studied for linear uncertain switched systems in infinite-time domain. Internal property of the systems is able to be illustrated from different perspectives with the help of above stability analysis. By employing uncertainty theory and the feature of switched systems, corresponding judgement theorems of these stabilities are proposed and verified. An example with respect to stability in measure is provided to display the validness of the results derived.
\end{abstract}

Keywords: Uncertain switched systems, Stability in measure, Almost sure stability, Stability in mean, Infinite-time domain.

\section{Introduction}

Switched systems[10] are a significant type of hybrid systems which include several sub-systems and a switching law orchestrating these sub-systems. Plenty of physical systems can be come down to switched systems in practical fields, such as electric power system[21], indoor temperature control system[1] and Robotic system[4]. From the 1990s, based on Lyapunov stability theory, many researchers began to investigate the stability of switched systems depending on the concrete form of sub-systems and the switching law. Branicky[3] employed multiple Lyapupov functions and iterated function systems theory as major tools to discuss the stability of switched systems. Hespanha and Morse[9] analyzed the exponential stability for switched systems by using an average dwell-time approach. In 2009, Lin and Antsaklis[11] considered the stability and stabilizability problems for a class of linear switched systems.

While switched systems are often influenced by external disturbances, therefore lots of mathematicians pay attention to stochastic switched systems[7] and their stability. Chatterjee and Liberzon[5] investigated the stability of a stochastic switched system through the method of multiple Lyapunov-like functions. Filipovic[8] proved the exponential $m$-stability for stochastic switched systems with the help of multiple Lyapunov functions on condition that the number of switches is finite in each bounded interval. In [2], input-to-state stability issue of nonlinear stochastic switched systems was handled with bounded disturbance input, and Lyapunov-like sufficient conditions were developed to guarantee the stability in $p$-th moment. In 2018, Ning et al.[18] used the semi-Markov kernel approach to analyze stability and stabilization of discrete-time stochastic switched systems when the sojourn time is lower and upper bounded.

Due to the fact that the real world and human behaviors are complex, the phenomena we encounter usually involve various forms of uncertainties. The majority of these uncertainties do not behave like randomness, such as the definition of overweight, the meaning of youth and the price of a new stock. In order to handle such uncertainties along an appropriate way, Liu[12] set up uncertainty theory by introducing uncertain measure, uncertain variable and uncertainty distribution. Furthermore, Liu[15] refined uncertainty theory to make it a branch of modern mathematics for modeling subjective uncertainty more accurately. It is worth mentioning, the concept of uncertain differential equation was introduced in [13]. From then on, uncertainty theory and

*Corresponding author. Email: 972718523@qq.com; 003012@nuist.edu.cn (Y. Shu).

${ }^{\dagger}$ Email: libnust@163.com (B. Li). 
its applications started to be extensively investigated, for example, portfolio selection model[30], resource extraction problem[25], production-inventory problem[20] and the like.

To illustrate the internal property of uncertain systems, the definition of stability in measure was proposed by Liu[14] in 2009, and then Yao et al.[28] obtained a sufficient condition to judge stability in measure of uncertain differential systems. In 2014, the concept of almost sure stability was introduced in [16] and a judgement criterion was established for this stability. Another stability named stability in mean was defined in [29] and the corresponding judgement theorem was presented for a family of uncertain systems, and its relationship with stability in measure was also derived. Then, Tao and Zhu[24] investigated stability and attractivity of the unique solution to an uncertain dynamic system under optimistic value criterion. In 2020, Liu and Zhang[17] considered stability in $p$-th moment of a special type of uncertain systems formulated by uncertain heat equations. These results are meaningful for revealing the internal property of uncertain systems from different aspects, and in this work three type of stabilities for linear uncertain switched systems will be profoundly studied in sequence.

The first contribution of this paper is that subjective uncertainty is considered in the stability analysis for switched systems, so that it has more applications than the switched systems mentioned in [11] and [5]. The second one is that three different stabilities of uncertain switched systems in infinite-time domain are analyzed deeply. Compared with the studies in many published papers such as [9] and [8], stability analysis in this work is more comprehensive and provides multiple perspectives to observe the internal property of switched systems. The third contribution is that, inspired by related articles [29, 23], three explicit theorems are proposed to judge stability in measure, almost sure stability and stability in mean. In brief, this work makes a contribution to broadening the horizon of stability analysis related to uncertain switched systems.

The rest of this paper is organized as follows: in the next section, basic concepts and theorems of uncertainty theory will be reviewed and linear uncertain switched systems in infinite-time domain will be introduced. In Section 3, an important lemma is presented, and then stability in measure of the systems will be researched by applying uncertain theory and the feature of switched systems. In Sections 4 and 5 , the sufficient conditions to guarantee almost sure stability and stability in mean are derived and testified for a class of linear uncertain switched systems, respectively. To clearly illustrate the internal property of uncertain switched systems, these three stabilities are very useful and convenient. At last, a numerical example is provided in Section 6 to show the effectiveness of the results in Section 3.

\section{Preliminary}

\section{$2.1 \quad$ Uncertainty Theory}

In convenience, some fundamental concepts and conclusions are introduced in this subsection. Let $\Gamma$ be a nonempty set, and $\mathcal{L}$ a $\sigma$-algebra over $\Gamma$. Each element $\Lambda \in \mathcal{L}$ is called an event.

Definition 2.1. [12] A set function $\mathcal{M}$ defined on the $\sigma$-algebra $\mathcal{L}$ is said to be an uncertain measure provided that it satisfies the following axioms:

Axiom 1. (Normality) $\mathcal{M}\{\Gamma\}=1$;

Axiom 2. (Self-Duality) $\mathcal{M}\{\Lambda\}+\mathcal{M}\left\{\Lambda^{c}\right\}=1$ for any event $\Lambda$;

Axiom 3. (Countable Subadditivity) $\mathcal{M}\left\{\bigcup_{i=1}^{\infty} \Lambda_{i}\right\} \leq \sum_{i=1}^{+\infty} \mathcal{M}\left\{\Lambda_{i}\right\}$ for any events $\Lambda_{1}, \Lambda_{2}, \cdots$

The triplet $(\Gamma, \mathcal{L}, \mathcal{M})$ is called an uncertainty space. Assume that $\left(\Gamma_{k}, \mathcal{L}_{k}, \mathcal{M}_{k}\right)$ are uncertainty spaces for $k=1,2, \cdots$, and $\mathcal{M}$ is a product uncertainty measure on $\sigma$-algebra $\mathcal{L}_{1} \times \mathcal{L}_{2} \times \cdots$, which satisfies the equality

$$
\mathcal{M}\left\{\prod_{k=1}^{+\infty} \Lambda_{k}\right\}=\bigwedge_{k=1}^{+\infty} \mathcal{M}_{k}\left\{\Lambda_{k}\right\}
$$

where $\Lambda_{k} \in \mathcal{L}_{k}$ for $k=1,2, \cdots$. 
Definition 2.2. [12] An uncertain variable is a measurable function $\xi$ from an uncertainty space $(\Gamma, \mathcal{L}, \mathcal{M})$ to the set of real numbers, namely, for any Borel set of real numbers, the set

$$
\{\xi \in B\}=\{\gamma \in \Gamma \mid \xi(\gamma) \in B\}
$$

is an event. And the uncertainty distribution $\Phi: \Re \rightarrow[0,1]$ of the uncertain variable $\xi$ is defined as

$$
\Phi(x)=\mathcal{M}\{\xi \leq x\} .
$$

Definition 2.3. [15] Let $\xi$ be an uncertain variable with regular uncertainty distribution $\Phi(x)$. Then the inverse function $\Phi^{-1}(\alpha)$ is called the inverse uncertainty distribution of $\xi$.

Definition 2.4. [12] Let $\xi$ be an uncertain variable. Then the expected value of $\xi$ is defined by

$$
E[\xi]=\int_{0}^{+\infty} \mathcal{M}\{\xi \geq r\} d r-\int_{-\infty}^{0} \mathcal{M}\{\xi \leq r\} d r
$$

provided that at least one of the two integrals is finite. The variance of $\xi$ is $V[\xi]=E\left[(\xi-e)^{2}\right]$ if the expected value of $\xi$ is a finite number $e$.

Based on the uncertainty space, Liu introduced the concepts of uncertain process, canonical process, uncertain differential equation, and etc.

Definition 2.5. [14] Let $T$ be an index set and let $(\Gamma, \mathcal{L}, \mathcal{M})$ be an uncertainty space. An uncertain process is a measurable function from $T \times(\Gamma, \mathcal{L}, \mathcal{M})$ to the set of real numbers, ie., for each $t \in T$ and any Borel set $B$ of real numbers, the set

$$
\left\{X_{t} \in B\right\}=\left\{\gamma \in \Gamma \mid X_{t}(\gamma) \in B\right\}
$$

is an event.

Definition 2.6. [14] An uncertain process $C_{t}$ is said to be a canonical process if

(i) $C_{0}=0$ and almost all sample paths are Lipschitz continuous,

(ii) $C_{t}$ has stationary and independent increments,

(iii) every increment $C_{s+t}-C_{s}$ is a normal uncertain variable with expected value 0 and variance $t^{2}$, whose uncertainty distribution is

$$
\Phi(x)=\left(1+\exp \left(\frac{-\pi x}{\sqrt{3} t}\right)\right)^{-1}, \quad x \in R .
$$

Theorem 2.1. [28] Let $C_{t}$ be a canonical process. Then there exists a nonnegative uncertain variable $K$ such that $K_{\gamma}$ is a Lipschitz constant of the sample path $C_{t}(\gamma)$ defined by:

$$
K_{\gamma}=\sup _{0 \leq t_{1} \leq t_{2}} \frac{C_{t_{2}}(\gamma)-C_{t_{1}}(\gamma)}{t_{2}-t_{1}}, \forall \gamma \in \Gamma
$$

satisfies

$$
\mathcal{M}\{K \leq x\} \geq 2\left(1+\exp \left(-\frac{\pi x}{\sqrt{3}}\right)\right)^{-1}-1
$$

which implies that

$$
\lim _{x \rightarrow+\infty} \mathcal{M}\{K \leq x\}=1 .
$$

Theorem 2.2. [6] Suppose that $C_{t}$ is a canonical process, and $X_{t}$ is an integrable uncertain process on $[a, b]$ with respect to $t$. Then the inequality

$$
\left|\int_{a}^{b} X_{t}(\gamma) d C_{t}(\gamma)\right| \leq K_{\gamma} \int_{a}^{b}\left|X_{t}(\gamma)\right| d t
$$

holds, where $K_{\gamma}$ is the Lipschitz constant of the sample path $C_{t}(\gamma)$. 
Definition 2.7. [13] Suppose $C_{t}$ is a canonical process, and $f$ and $g$ are given functions. Then

$$
d X_{t}=f\left(X_{t}, t\right) d t+g\left(X_{t}, t\right) d C_{t}
$$

is called an uncertain differential equation. A solution is an uncertain process $X_{t}$ that satisfies the following uncertain integral equation:

$$
X_{t}=X_{t_{0}}+\int_{t_{0}}^{t} f\left(X_{s}, s\right) d s+\int_{t_{0}}^{t} g\left(X_{s}, s\right) d C_{s} .
$$

Definition 2.8. [27] Let $\alpha$ be a real number with $0<\alpha<1$. An uncertain differential equation

$$
d X_{t}=f\left(X_{t}, t\right) d t+g\left(X_{t}, t\right) d C_{t}
$$

is said to have an $\alpha$-path $X_{t}^{\alpha}$ if it solves the corresponding ordinary differential equation

$$
d X_{t}^{\alpha}=f\left(X_{t}^{\alpha}, t\right) d t+\left|g\left(X_{t}^{\alpha}, t\right)\right| \Phi^{-1}(\alpha) d t
$$

where $\Phi^{-1}(\alpha)$ is the inverse standard normal uncertainty distribution, i.e.,

$$
\Phi^{-1}(\alpha)=\frac{\sqrt{3}}{\pi} \ln \frac{1-\alpha}{\alpha} .
$$

Definition 2.9. [26] Suppose that $\boldsymbol{C}_{t}$ is an m-dimensional canonical process, $\boldsymbol{f}(t, \boldsymbol{x})$ is a vector-valued function from $T \times R^{n}$ to $R^{n}$, and $\boldsymbol{g}(t, \boldsymbol{x})$ is a matrix-valued function from $T \times R^{n}$ to the set of $n \times m$ matrices. Then

$$
d \boldsymbol{X}_{t}=\boldsymbol{f}\left(t, \boldsymbol{X}_{t}\right) d t+\boldsymbol{g}\left(t, \boldsymbol{X}_{t}\right) d \boldsymbol{C}_{t}
$$

is called a multi-dimensional uncertain differential equation driven by an $m$-dimensional canonical process. And an $n$-dimensional uncertain process $\boldsymbol{X}_{\boldsymbol{t}}$ that satisfies the corresponding uncertain integral equation is called a solution of the uncertain differential equation.

\subsection{Linear Uncertain Switched Systems}

Actually, an uncertain switched system introduced in this subsection is a switched system perturbed by an uncertain process. A linear uncertain switched system in infinite-time domain written as uncertain differential equations will be considered in the following:

$$
\left\{\begin{array}{l}
d \boldsymbol{X}_{t}=A_{i(k)}(t) \boldsymbol{X}_{t} d t+B_{i(k)}(t) \boldsymbol{X}_{t} d C_{t}, \quad t \in[0,+\infty) \\
\quad i(k) \in I=\{1,2, \cdots, M\}, \\
\left.\boldsymbol{X}\right|_{t=0}=\boldsymbol{X}_{0},
\end{array}\right.
$$

where $\boldsymbol{X}_{t} \in R^{n}$ represents the state vector of the system, the matrix functions $A_{i(k)}:[0,+\infty) \rightarrow R^{n \times n}$ and $B_{i(k)}:[0,+\infty) \rightarrow R^{n \times m}$ are bounded for any $i(k) \in I$, and $C_{t}$ is a canonical process defined on an uncertainty space, representing the noise of the system. Throughout this paper, for a matrix $A=\left[a_{i j}\right]_{m \times n}$ and a vector $\boldsymbol{X}=\left(x_{1}, x_{2}, \cdots, x_{n}\right)^{T}, L 1-$ norm is employed to measure them as follows:

$$
\|A\|=\max _{j} \sum_{i=1}^{m}\left|a_{i j}\right|, \quad\|\boldsymbol{X}\|=\sum_{i=1}^{n}\left|x_{i}\right| .
$$

The switching law of uncertain switched system (1) defined on the interval $[0,+\infty)$ is

$$
\Lambda=\left(\left(t_{0}, i(0)\right),\left(t_{1}, i(1)\right), \cdots,\left(t_{N}, i(N)\right), \cdots\right),
$$

where $t_{k}(k=0,1, \cdots, N, \cdots)$ stand for the switching instants, and $0=t_{0}<t_{1}<\cdots<t_{N}<\cdots<+\infty$. The tuple $\left(t_{k}, i(k)\right)$ means that at the instant $t_{k}$ the system switches to sub-system $i(k)$ from sub-system $i(k-1)$, that is, sub-system $i(k)$ alone keeps active in time interval $\left[t_{k}, t_{k+1}\right)$ for each $k \in\{0,1, \cdots, N, \cdots\}$. 
The symbols $\|A(t)\|$ and $\|B(t)\|$ are adopted to represent the supremum of two sequences of bounded functions $\left\|A_{i(k)}(t)\right\|$ and $\left\|B_{i(k)}(t)\right\|(i(k)=0,1, \cdots, M)$, respectively. Consequently, the following expressions are established for $t \in[0,+\infty)$ :

$$
\begin{aligned}
& \|A(t)\|=\sup _{i(k)}\left\{\left\|A_{i(k)}(t)\right\| \mid i(k)=0,1, \cdots, M\right\}, \\
& \|B(t)\|=\sup _{i(k)}\left\{\left\|B_{i(k)}(t)\right\| \mid i(k)=0,1, \cdots, M\right\} .
\end{aligned}
$$

\section{$3 \quad$ Stability in Measure}

In this section, stability in measure of uncertain switched systems will be analyzed according to uncertainty theory and the feature of switched systems. Before the investigation on this stability, a lemma about vector functions is firstly presented.

Lemma 3.1. If a vector function $\boldsymbol{f}(t)=\left(f_{1}(t), f_{2}(t), \cdots, f_{n}(t)\right)^{T}$ is differentiable when $t>0$, then $\|\boldsymbol{f}(t)\|$ is differentiable almost everywhere in the interval $(0,+\infty)$.

Proof. Based on the condition known, we obtain that

$$
\left|f_{1}(t)\right|= \begin{cases}f_{1}(t), & \text { if } t \in f_{1}^{-1}(0,+\infty) \\ 0, & \text { if } t \in f_{1}^{-1}\{0\} \\ -f_{1}(t), & \text { if } t \in f_{1}^{-1}(-\infty, 0),\end{cases}
$$

namely, $\left|f_{1}(t)\right|$ is differentiable on open sets $f_{1}^{-1}(0,+\infty)$ and $f_{1}^{-1}(-\infty, 0)$. Denote $W=f_{1}^{-1}\{0\}$ which is a closed set. For any $\varepsilon>0$, there exists an open set $V(\varepsilon) \subseteq W$, such that

$$
m(W-V(\varepsilon))<\varepsilon,
$$

where $m(\cdot)$ stands for Lebesgue measure. It is not different to know that $\left|f_{1}(t)\right|$ is differentiable on the open set $\underset{\varepsilon>0}{\cup} V(\varepsilon)$, since we have

$$
\left\{t \in(0,+\infty)|| f_{1}(t) \mid \text { is not differentiable. }\right\} \subseteq W-\underset{\varepsilon>0}{\cup} V(\varepsilon),
$$

and $m\left(W-\cup_{\varepsilon>0} V(\varepsilon)\right) \leq m(W-V(\varepsilon))<\varepsilon$ which implies that $m\left(W-\cup_{\varepsilon>0} V(\varepsilon)\right)=0$. Therefore the Lebesgue measure of the set $\left\{t \in(0,+\infty)|| f_{1}(t) \mid\right.$ is not differentiable. $\}$ is 0 . In the same way, the equalities

$$
m\left(\left\{t \in(0,+\infty)|| f_{i}(t) \mid \text { is not differentiable. }\right\}\right)=0
$$

are derived for $i=2,3, \cdots, n$.

Because $\|\boldsymbol{f}(t)\|=\sum_{i=1}^{n}\left|f_{i}(t)\right|$, the following equality is easily obtained:

$$
m(\{t \in(0,+\infty) \mid\|\boldsymbol{f}(t)\| \text { is not differentiable. }\})=0 .
$$

This completes the proof.

Inspired by the related papers[11, 28], stability in measure of uncertain switched system (1) will be deeply discussed in the following.

Definition 3.1. An uncertain matrix differential equation is said to be stable in measure if for any solutions $\boldsymbol{X}_{t}$ and $\boldsymbol{Y}_{t}$ with initial values $\boldsymbol{X}_{0}$ and $\boldsymbol{Y}_{0}$, respectively, we have

$$
\lim _{\left\|\boldsymbol{X}_{0}-\boldsymbol{Y}_{0}\right\| \rightarrow 0} \mathcal{M}\left\{\sup _{t \geq 0}\left\|\boldsymbol{X}_{t}-\boldsymbol{Y}_{t}\right\| \leq \varepsilon\right\}=1
$$

for any given number $\varepsilon>0$. 
Theorem 3.1. Uncertain switched system (1) is stable in measure if the supremum of two sequences of functions $\left\|A_{i(k)}(t)\right\|$ and $\left\|B_{i(k)}(t)\right\|(i(k)=0,1, \cdots, M)$ are both integrable on $[0,+\infty)$, namely,

$$
\int_{0}^{+\infty}\|A(t)\| d t<+\infty, \quad \int_{0}^{+\infty}\|B(t)\| d t<+\infty .
$$

Proof. According to uncertain differential equations in system (1), $\boldsymbol{X}_{t}(\gamma)-\boldsymbol{Y}_{t}(\gamma)$ is differentiable for each $\gamma \in \Gamma$ when $t \in(0,+\infty)-\left\{t_{1}, t_{2}, \cdots, t_{N}, \cdots\right\}$. Obviously, Lebesgue measure of the set $\left\{t_{1}, t_{2}, \cdots, t_{N}, \cdots\right\}$ consisting of switching instants is 0 , so $\left\|\boldsymbol{X}_{t}(\gamma)-\boldsymbol{Y}_{t}(\gamma)\right\|$ is differentiable almost everywhere on the interval $(0,+\infty)$ according to Lemma 3.1.

Denote $A_{\gamma}=\left\{t \in(0,+\infty) \mid\left\|\boldsymbol{X}_{t}(\gamma)-\boldsymbol{Y}_{t}(\gamma)\right\|\right.$ is differentiable. $\}$, and $B_{\gamma}=(0,+\infty)-A_{\gamma}$. It is easy to know that $m\left(B_{\gamma}\right)=0$. Then write $\boldsymbol{X}_{t}(\gamma)=\left(x_{1}(\gamma), x_{2}(\gamma), \cdots, x_{n}(\gamma)\right)^{T}$ and $Y_{t}(\gamma)=\left(y_{1}(\gamma), y_{2}(\gamma), \cdots, y_{n}(\gamma)\right)^{T}$.

For any $t \in A_{\gamma}$, there exists an interval $\left[t_{k}, t_{k+1}\right)$ such that it includes the moment $t$, since, based on Theorem 2.2, we obtain that

$$
\begin{aligned}
d\left\|\boldsymbol{X}_{t}(\gamma)-\boldsymbol{Y}_{t}(\gamma)\right\| & =d \sum_{i=1}^{n}\left|x_{i}(\gamma)-y_{i}(\gamma)\right| \\
& =d \sum_{i=1}^{n} \pm\left(x_{i}(\gamma)-y_{i}(\gamma)\right) \\
& =\sum_{i=1}^{n} \pm\left(d x_{i}(\gamma)-d y_{i}(\gamma)\right) \\
& \leq \sum_{i=1}^{n}\left|d x_{i}(\gamma)-d y_{i}(\gamma)\right| \\
& =\left\|d \boldsymbol{X}_{t}(\gamma)-d \boldsymbol{Y}_{t}(\gamma)\right\| \\
& \leq\left\|A_{i(k)}(t)\left(\boldsymbol{X}_{t}(\gamma)-\boldsymbol{Y}_{t}(\gamma)\right) d t+B_{i(k)}(t)\left(\boldsymbol{X}_{t}(\gamma)-\boldsymbol{Y}_{t}(\gamma)\right) d C_{t}(\gamma)\right\| \\
& \leq\left\|A_{i(k)}(t)\left(\boldsymbol{X}_{t}(\gamma)-\boldsymbol{Y}_{t}(\gamma)\right) d t\right\|+\left\|B_{i(k)}(t)\left(\boldsymbol{X}_{t}(\gamma)-\boldsymbol{Y}_{t}(\gamma)\right) d C_{t}(\gamma)\right\| \\
& \leq\left\|A_{i(k)}(t)\right\| \cdot\left\|\left(\boldsymbol{X}_{t}(\gamma)-\boldsymbol{Y}_{t}(\gamma)\right)\right\| d t+\left\|B_{i(k)}(t)\right\| \cdot\left\|\left(\boldsymbol{X}_{t}(\gamma)-\boldsymbol{Y}_{t}(\gamma)\right) d C_{t}(\gamma)\right\| \\
& \leq\left\|A_{i(k)}(t)\right\| \cdot\left\|\left(\boldsymbol{X}_{t}(\gamma)-\boldsymbol{Y}_{t}(\gamma)\right)\right\| d t+K_{\gamma}\left\|B_{i(k)}(t)\right\| \cdot\left\|\left(\boldsymbol{X}_{t}(\gamma)-\boldsymbol{Y}_{t}(\gamma)\right)\right\| d t,
\end{aligned}
$$

where $K_{\gamma}$ is the Lipschitz constant of the sample path $C_{t}(\gamma)$ in Theorem 2.1. It follows that

$$
\left\|\boldsymbol{X}_{t}(\gamma)-\boldsymbol{Y}_{t}(\gamma)\right\| \leq\left\|\boldsymbol{X}_{t_{k}}(\gamma)-\boldsymbol{Y}_{t_{k}}(\gamma)\right\| \cdot \exp \left(\int_{t_{k}}^{t}\left\|A_{i(k)}(s)\right\| d s\right) \cdot \exp \left(K_{\gamma} \int_{t_{k}}^{t}\left\|B_{i(k)}(s)\right\| d s\right) .
$$

For each $t \in B_{\gamma}$, we can select $t_{1}^{\prime} \in\left(\left(1-\frac{1}{2}\right) t, t\right)$ such that $t_{1}^{\prime} \in A_{\gamma}$, and then it is easy to find $t_{2}^{\prime} \in$ $\left(\left(1-\frac{1}{3}\right) t, t\right)-\left\{t_{1}^{\prime}\right\}$ such that $t_{2}^{\prime} \in A_{\gamma}$. Similarly, for any $n \in N_{+}$, we select $t_{n}^{\prime} \in\left(\left(1-\frac{1}{n+1}\right) t, t\right)-\left\{t_{1}^{\prime}, t_{2}^{\prime}, \ldots, t_{n-1}^{\prime}\right\}$ such that $t_{n}^{\prime} \in A_{\gamma}$. Apparently, we know that $t_{n}^{\prime} \rightarrow t$, as $n \rightarrow+\infty$. For any $n \in N_{+}, t_{n}^{\prime} \in A_{\gamma}$, according to previous deduction, the following inequality is obtained:

$$
\begin{aligned}
\left\|\boldsymbol{X}_{t_{n}^{\prime}}(\gamma)-\boldsymbol{Y}_{t_{n}^{\prime}}(\gamma)\right\| & \leq\left\|\boldsymbol{X}_{t_{k}}(\gamma)-\boldsymbol{Y}_{t_{k}}(\gamma)\right\| \cdot \exp \left(\int_{t_{k}}^{t_{n}^{\prime}}\left\|A_{i(k)}(s)\right\| d s\right) \cdot \exp \left(K_{\gamma} \int_{t_{k}}^{t_{n}^{\prime}}\left\|B_{i(k)}(s)\right\| d s\right) \\
& \leq\left\|\boldsymbol{X}_{t_{k}}(\gamma)-\boldsymbol{Y}_{t_{k}}(\gamma)\right\| \cdot \exp \left(\int_{t_{k}}^{t}\left\|A_{i(k)}(s)\right\| d s\right) \cdot \exp \left(K_{\gamma} \int_{t_{k}}^{t}\left\|B_{i(k)}(s)\right\| d s\right) .
\end{aligned}
$$

Let $n \rightarrow+\infty$, because $X_{t}$ is sample-continuous for the event $\gamma$, we have

$$
\left\|\boldsymbol{X}_{t}(\gamma)-\boldsymbol{Y}_{t}(\gamma)\right\| \leq\left\|\boldsymbol{X}_{t_{k}}(\gamma)-\boldsymbol{Y}_{t_{k}}(\gamma)\right\| \cdot \exp \left(\int_{t_{k}}^{t}\left\|A_{i(k)}(s)\right\| d s\right) \cdot \exp \left(K_{\gamma} \int_{t_{k}}^{t}\left\|B_{i(k)}(s)\right\| d s\right)
$$


To sum up, for any $t \in(0,+\infty)$, combining with the arbitrariness of $\gamma$, the inequality

$$
\left\|\boldsymbol{X}_{t}-\boldsymbol{Y}_{t}\right\| \leq\left\|\boldsymbol{X}_{t_{k}}-\boldsymbol{Y}_{t_{k}}\right\| \cdot \exp \left(\int_{t_{k}}^{t}\left\|A_{i(k)}(s)\right\| d s\right) \cdot \exp \left(K \int_{t_{k}}^{t}\left\|B_{i(k)}(s)\right\| d s\right)
$$

is almost surely satisfied, where $K$ is a nonnegative uncertain variable such that

$$
\lim _{x \rightarrow+\infty} \mathcal{M}\{\gamma \in \Gamma \mid K(\gamma) \leq x\}=1
$$

in accordance with Theorem 2.1. In order to make connections with initial states, the features of switched system (1) have to be applied, and then based on the definition of $\|A(t)\|$ and $\|B(t)\|$, we get that

$$
\begin{aligned}
\left\|\boldsymbol{X}_{t}-\boldsymbol{Y}_{t}\right\| \leq & \left\|\boldsymbol{X}_{t_{k}}-\boldsymbol{Y}_{t_{k}}\right\| \cdot \exp \left(\int_{t_{k}}^{t}\left\|A_{i(k)}(s)\right\| d s\right) \cdot \exp \left(K \int_{t_{k}}^{t}\left\|B_{i(k)}(s)\right\| d s\right) \\
\leq & \left\|\boldsymbol{X}_{t_{k-1}}-\boldsymbol{Y}_{t_{k-1}}\right\| \cdot \exp \left(\int_{t_{k-1}}^{t_{k}}\left\|A_{i(k-1)}(s)\right\| d s\right) \cdot \exp \left(K \int_{t_{k-1}}^{t_{k}}\left\|B_{i_{(k-1)}}(s)\right\| d s\right) \\
& \cdot \exp \left(\int_{t_{k}}^{t}\left\|A_{i(k)}(s)\right\| d s\right) \cdot \exp \left(K \int_{t_{k}}^{t}\left\|B_{i(k)}(s)\right\| d s\right) \\
\leq & \left\|\boldsymbol{X}_{t_{0}}-\boldsymbol{Y}_{t_{0}}\right\| \cdot \exp \left(\sum_{j=0}^{k-1} \int_{t_{j}}^{t_{j+1}}\left\|A_{i(j)}(s)\right\| d s\right) \cdot \exp \left(K \sum_{j=0}^{k-1} \int_{t_{j}}^{t_{j+1}}\left\|B_{i(j)}(s)\right\| d s\right) \\
& \cdot \exp \left(\int_{t_{k}}^{t}\left\|A_{i(k)}(s)\right\| d s\right) \cdot \exp \left(K \int_{t_{k}}^{t}\left\|B_{i(k)}(s)\right\| d s\right) \\
\leq & \left\|\boldsymbol{X}_{0}-\boldsymbol{Y}_{0}\right\| \cdot \exp \left(\sum_{j=0}^{k-1} \int_{t_{j}}^{t_{j+1}}\|A(s)\| d s\right) \cdot \exp \left(K \sum_{j=0}^{k-1} \int_{t_{j}}^{t_{j+1}}\|B(s)\| d s\right) \\
& \cdot \exp \left(\int_{t_{k}}^{t}\|A(s)\| d s\right) \cdot \exp \left(K \int_{t_{k}}^{t}\|B(s)\| d s\right) \\
= & \left\|\boldsymbol{X}_{0}-\boldsymbol{Y}_{0}\right\| \cdot \exp \left(\int_{0}^{t}\|A(s)\| d s\right) \cdot \exp \left(K \int_{0}^{t}\|B(s)\| d s\right) \\
\leq & \left\|\boldsymbol{X}_{0}-\boldsymbol{Y}_{0}\right\| \cdot \exp \left(\int_{0}^{+\infty}\|A(s)\| d s\right) \cdot \exp \left(K \int_{0}^{+\infty}\|B(s)\| d s\right) \\
= & \left\|\boldsymbol{X}_{0}-\boldsymbol{Y}_{0}\right\| \cdot \exp \left(\int_{0}^{+\infty}(\|A(s)\|+K\|B(s)\|) d s\right)
\end{aligned}
$$

is almost surely true, which implies that

$$
\sup _{t>0}\left\|\boldsymbol{X}_{t}-\boldsymbol{Y}_{t}\right\| \leq\left\|\boldsymbol{X}_{0}-\boldsymbol{Y}_{0}\right\| \cdot \exp \left(\int_{0}^{+\infty}(\|A(s)\|+K\|B(s)\|) d s\right)
$$

almost surely. According to Equation (6), for any given $\epsilon>0$, there exists a positive number $H$ such that

$$
\mathcal{M}\{\gamma \in \Gamma \mid K(\gamma) \leq H\}>1-\epsilon
$$

For any given number $\varepsilon>0$, we take

$$
\delta=\exp \left(-\int_{0}^{+\infty}(\|A(s)\|+H\|B(s)\|) d s\right) \varepsilon .
$$

Under the conditions $\left\|\boldsymbol{X}_{0}-\boldsymbol{Y}_{0}\right\| \leq \delta$ and $K(\gamma) \leq H$, the inequality $\sup _{t>0}\left\|\boldsymbol{X}_{t}(\gamma)-\boldsymbol{Y}_{t}(\gamma)\right\| \leq \varepsilon$ is true according to Inequality (8). This result indicates that

$$
\mathcal{M}\left\{\sup _{t>0} \mid \boldsymbol{X}_{t}-\boldsymbol{Y}_{t} \| \leq \varepsilon\right\} \geq \mathcal{M}\{\gamma \in \Gamma \mid K(\gamma) \leq H\}>1-\epsilon,
$$


provided that $\left\|\boldsymbol{X}_{0}-\boldsymbol{Y}_{0}\right\| \leq \delta$. That is to say, the following equality is satisfied:

$$
\lim _{\left\|\boldsymbol{X}_{0}-\boldsymbol{Y}_{0}\right\| \rightarrow 0} \mathcal{M}\left\{\sup _{t>0}\left\|\boldsymbol{X}_{t}-\boldsymbol{Y}_{t}\right\| \leq \varepsilon\right\}=1 .
$$

Obviously, we know that

$$
\lim _{\left\|\boldsymbol{X}_{0}-\boldsymbol{Y}_{0}\right\| \rightarrow 0} \mathcal{M}\left\{\left\|\boldsymbol{X}_{0}-\boldsymbol{Y}_{0}\right\| \leq \varepsilon\right\}=1 .
$$

Combining these two equalities, it is easy to obtain

$$
\lim _{\left\|\boldsymbol{X}_{0}-\boldsymbol{Y}_{0}\right\| \rightarrow 0} \mathcal{M}\left\{\sup _{t \geq 0}\left\|\boldsymbol{X}_{t}-\boldsymbol{Y}_{t}\right\| \leq \varepsilon\right\}=1 .
$$

Therefore, uncertain switched system (1) is stable in measure. This completes the proof.

\section{Almost sure stability}

Almost sure stability of uncertain systems was defined and studied in [16], based on this investigation we analyze such stability with respect to uncertain switched system (1).

Definition 4.1. An uncertain matrix differential equation is said to be almost surely stable if for any solutions $\boldsymbol{X}_{t}$ and $\boldsymbol{Y}_{t}$ with initial values $X_{0}$ and $Y_{0}$, respectively, we have

$$
\mathcal{M}\left\{\lim _{\left\|\boldsymbol{X}_{0}-\boldsymbol{Y}_{0}\right\| \rightarrow 0} \sup _{t \geq 0}\left\|\boldsymbol{X}_{t}-\boldsymbol{Y}_{t}\right\|=0\right\}=1 .
$$

Theorem 4.1. System (1) is almost surely stable provided that the supremum of the functions $\left\|A_{i(k)}(t)\right\|$ and $\left\|B_{i(k)}(t)\right\|(i(k)=0,1, \cdots, M)$ are both integrable on $[0,+\infty)$, that is,

$$
\int_{0}^{+\infty}\|A(t)\| d t<+\infty, \quad \int_{0}^{+\infty}\|B(t)\| d t<+\infty .
$$

Proof. According to Inequality (5), for any $t \in(0,+\infty)$, because of the arbitrariness of $\gamma$, we always have

$$
\left\|\boldsymbol{X}_{t}-\boldsymbol{Y}_{t}\right\| \leq\left\|\boldsymbol{X}_{t_{k}}-\boldsymbol{Y}_{t_{k}}\right\| \cdot \exp \left(\int_{t_{k}}^{t}\left\|A_{i(k)}(s)\right\| d s\right) \cdot \exp \left(K \int_{t_{k}}^{t}\left\|B_{i(k)}(s)\right\| d s\right)
$$

almost surely, where $K$ is a nonnegative uncertain variable such that

$$
\lim _{x \rightarrow+\infty} \mathcal{M}\{\gamma \in \Gamma \mid K(\gamma) \leq x\}=1
$$

according to Theorem 2.1. Similar with the method in the proof of Theorem 3.1, the following inequality

$$
\begin{aligned}
\left\|\boldsymbol{X}_{t}-\boldsymbol{Y}_{t}\right\| & \leq\left\|\boldsymbol{X}_{t_{k}}-\boldsymbol{Y}_{t_{k}}\right\| \cdot \exp \left(\int_{t_{k}}^{t}\left\|A_{i(k)}(s)\right\| d s\right) \cdot \exp \left(K \int_{t_{k}}^{t}\left\|B_{i(k)}(s)\right\| d s\right) \\
& \leq\left\|\boldsymbol{X}_{0}-\boldsymbol{Y}_{0}\right\| \cdot \exp \left(\int_{0}^{+\infty}(\|A(s)\|+K\|B(s)\|) d s\right)
\end{aligned}
$$

is almost surely true when $t>0$, which indicates that

$$
\sup _{t>0}\left\|\boldsymbol{X}_{t}-\boldsymbol{Y}_{t}\right\| \leq\left\|\boldsymbol{X}_{0}-\boldsymbol{Y}_{0}\right\| \cdot \exp \left(\int_{0}^{+\infty}(\|A(s)\|+K\|B(s)\|) d s\right)
$$

almost surely. In terms of Equation (10), for any given $\varepsilon>0$, there exists a positive number $H$ such that

$$
\mathcal{M}\{\gamma \in \Gamma \mid K(\gamma) \leq H\}>1-\varepsilon
$$


Denote

$$
\delta=\exp \left(-\int_{0}^{+\infty}(\|A(s)\|+H\|B(s)\|) d s\right) \varepsilon .
$$

Then $\sup _{t>0}\left\|\boldsymbol{X}_{t}(\gamma)-\boldsymbol{Y}_{t}(\gamma)\right\| \leq \varepsilon$ provided that $\left\|\boldsymbol{X}_{0}-\boldsymbol{Y}_{0}\right\| \leq \delta$ and $K(\gamma) \leq H$, that is, the following equality

$$
\lim _{\left\|\boldsymbol{X}_{0}-\boldsymbol{Y}_{0}\right\| \rightarrow 0} \sup _{t>0}\left\|\boldsymbol{X}_{t}-\boldsymbol{Y}_{t}\right\|=0
$$

is satisfied when $K(\gamma) \leq H$. Hence we get that

$$
\mathcal{M}\left\{\lim _{\left\|\boldsymbol{X}_{0}-\boldsymbol{Y}_{0}\right\| \rightarrow 0} \sup _{t>0} \mid \boldsymbol{X}_{t}-\boldsymbol{Y}_{t} \|=0\right\} \geq \mathcal{M}\{\gamma \in \Gamma \mid K(\gamma) \leq H\}>1-\varepsilon,
$$

which means

$$
\mathcal{M}\left\{\lim _{\left\|\boldsymbol{X}_{0}-\boldsymbol{Y}_{0}\right\| \rightarrow 0} \sup _{t>0}\left\|\boldsymbol{X}_{t}-\boldsymbol{Y}_{t}\right\|=0\right\}=1
$$

Obviously, we know that

$$
\mathcal{M}\left\{\lim _{\left\|\boldsymbol{X}_{0}-\boldsymbol{Y}_{0}\right\| \rightarrow 0}\left\|\boldsymbol{X}_{0}-\boldsymbol{Y}_{0}\right\|=0\right\}=1
$$

Combining the above two equalities, the following result is obtained:

$$
\mathcal{M}\left\{\lim _{\left\|\boldsymbol{X}_{0}-\boldsymbol{Y}_{0}\right\| \rightarrow 0} \sup _{t \geq 0}\left\|\boldsymbol{X}_{t}-\boldsymbol{Y}_{t}\right\|=0\right\}=1
$$

In conclusion, system (1) is almost surely stable according to Definition 4.1. This theorem is proved.

\section{Stability in Mean}

Investigation on stability in mean of uncertain systems was initiated in [29], and then we consider stability in mean of uncertain switched system (1) in this section.

Definition 5.1. An uncertain matrix differential equation is said to be stable in mean if for any solutions $\boldsymbol{X}_{t}$ and $\boldsymbol{Y}_{t}$ with initial values $\boldsymbol{X}_{0}$ and $\boldsymbol{Y}_{0}$, respectively, we have

$$
\lim _{\left\|\boldsymbol{X}_{0}-\boldsymbol{Y}_{0}\right\| \rightarrow 0} E\left[\sup _{t \geq 0}\left\|\boldsymbol{X}_{t}-\boldsymbol{Y}_{t}\right\|\right]=0
$$

Theorem 5.1. If the supremum of a sequence of bounded functions $\left\|A_{i(k)}(t)\right\|(i(k)=0,1, \cdots, M)$ is integrable on $[0,+\infty)$, and the integral of the supremum of $\left\{\left\|B_{i(k)}(t)\right\| \mid i(k)=0,1, \cdots, M\right\}$ on $[0,+\infty)$ is smaller than $\frac{\pi}{\sqrt{3}}$, namely,

$$
\int_{0}^{+\infty}\|A(t)\| d t<+\infty, \quad \int_{0}^{+\infty}\|B(t)\| d t<\frac{\pi}{\sqrt{3}}
$$

then system (1) is stable in mean.

Proof. Based on Inequality (5) in Section 3, for any $t \in(0,+\infty)$, because event $\gamma$ is arbitrary, we have

$$
\left\|\boldsymbol{X}_{t}-\boldsymbol{Y}_{t}\right\| \leq\left\|\boldsymbol{X}_{t_{k}}-\boldsymbol{Y}_{t_{k}}\right\| \cdot \exp \left(\int_{t_{k}}^{t}\left\|A_{i(k)}(s)\right\| d s\right) \cdot \exp \left(K \int_{t_{k}}^{t}\left\|B_{i(k)}(s)\right\| d s\right)
$$

almost surely, where $K$ is a nonnegative uncertain variable such that

$$
\mathcal{M}\{K(\gamma) \geq x\}=1-\mathcal{M}\{K(\gamma)<x\} \leq 2\left(1+\exp \left(\frac{\pi x}{\sqrt{3}}\right)\right)^{-1}
$$


according to Theorem 2.1. Employing the same approach in the proof of Theorem 3.1, we deduce that

$$
\begin{aligned}
\left\|\boldsymbol{X}_{t}-\boldsymbol{Y}_{t}\right\| & \leq\left\|\boldsymbol{X}_{t_{k}}-\boldsymbol{Y}_{t_{k}}\right\| \cdot \exp \left(\int_{t_{k}}^{t}\left\|A_{i(k)}(s)\right\| d s\right) \cdot \exp \left(K \int_{t_{k}}^{t}\left\|B_{i(k)}(s)\right\| d s\right) \\
& \leq\left\|\boldsymbol{X}_{0}-\boldsymbol{Y}_{0}\right\| \cdot \exp \left(\int_{0}^{+\infty}\|A(s)\| d s\right) \cdot \exp \left(K \int_{0}^{+\infty}\|B(s)\| d s\right)
\end{aligned}
$$

is almost surely true, which implies that

$$
\sup _{t>0}\left\|\boldsymbol{X}_{t}-\boldsymbol{Y}_{t}\right\| \leq\left\|\boldsymbol{X}_{0}-\boldsymbol{Y}_{0}\right\| \cdot \exp \left(\int_{0}^{+\infty}\|A(s)\| d s\right) \cdot \exp \left(K \int_{0}^{+\infty}\|B(s)\| d s\right)
$$

almost surely. Taking expected value on both sides of Inequality (12), then the following inequality

$$
E\left[\sup _{t>0}\left\|\boldsymbol{X}_{t}-\boldsymbol{Y}_{t}\right\|\right] \leq\left\|\boldsymbol{X}_{0}-\boldsymbol{Y}_{0}\right\| \cdot \exp \left(\int_{0}^{+\infty}\|A(s)\| d s\right) \cdot E\left[\exp \left(K \int_{0}^{+\infty}\|B(s)\| d s\right)\right]
$$

is obtained. Since the bounded function $\|A(t)\|$ is integrable on $[0,+\infty)$, we easily get

$$
\exp \left(\int_{0}^{+\infty}\|A(s)\| d s\right)<+\infty \text {. }
$$

For the expected value

$$
E\left[\exp \left(K \int_{0}^{+\infty}\|B(s)\| d s\right)\right]
$$

we denote that

$$
l=\int_{0}^{+\infty}\|B(s)\| d s
$$

It follows from Definition 2.4 of expected value and Theorem 2.1 that

$$
\begin{aligned}
E\left[\exp \left(K \int_{0}^{+\infty}\|B(s)\| d s\right)\right] & =E[\exp (l K)] \\
& =\int_{0}^{+\infty} \mathcal{M}\{\exp (l K) \geq x\} d x \\
& =\int_{0}^{+\infty} \mathcal{M}\left\{K \geq \frac{\ln x}{l}\right\} d x \\
& \leq 2 \int_{0}^{+\infty}\left(1+\exp \left(\frac{\pi \ln x}{\sqrt{3} l}\right)\right)^{-1} d x \\
& =2 \int_{0}^{+\infty}\left(1+x^{\frac{\pi}{\sqrt{3} l}}\right)^{-1} d x<+\infty .
\end{aligned}
$$

Combining with Inequality (13), the following equality is obtained:

$$
\lim _{\left\|\boldsymbol{X}_{0}-\boldsymbol{Y}_{0}\right\| \rightarrow 0} E\left[\sup _{t>0}\left\|\boldsymbol{X}_{t}-\boldsymbol{Y}_{t}\right\|\right]=0
$$

When $t=0$, we apparently know that

$$
\lim _{\left\|\boldsymbol{X}_{0}-\boldsymbol{Y}_{0}\right\| \rightarrow 0} E\left[\left\|\boldsymbol{X}_{0}-\boldsymbol{Y}_{0}\right\|\right]=\lim _{\left\|\boldsymbol{X}_{0}-\boldsymbol{Y}_{0}\right\| \rightarrow 0}\left\|\boldsymbol{X}_{0}-\boldsymbol{Y}_{0}\right\|=0 .
$$

Combining the above two equalities, we get

$$
\lim _{\left\|\boldsymbol{X}_{0}-\boldsymbol{Y}_{0}\right\| \rightarrow 0} E\left[\sup _{t \geq 0}\left\|\boldsymbol{X}_{t}-\boldsymbol{Y}_{t}\right\|\right]=0 .
$$

Hence the conclusion of the theorem is demonstrated, and the proof has been completed. 


\section{$6 \quad$ Numerical Example}

In order to illustrate the validness of Theorem 3.1, an example concerning stability in measure will be provided. Now we consider the following uncertain switched system:

$$
\left\{\begin{array}{l}
d \boldsymbol{X}_{t}=A_{i(k)}(t) \boldsymbol{X}_{t} d t+B_{i(k)}(t) \boldsymbol{X}_{t} d C_{t}, \quad t \in[0,+\infty) \\
\quad i(k) \in I=\{1,2, \cdots, 5\} \\
\boldsymbol{X}_{0}=(0.5,0.5)^{T}
\end{array}\right.
$$

where $\boldsymbol{X}_{t}=\left(x_{1}(t), x_{2}(t)\right)^{T} \in R^{2}$ represents the state vector of the system, and

$$
\begin{aligned}
& A_{1}(t)=\left[\begin{array}{cc}
e^{-t} & \frac{5}{1+t^{2}} \\
\frac{2}{1+t^{2}} & e^{-\frac{t}{4}}
\end{array}\right], \quad A_{2}(t)=\left[\begin{array}{cc}
e^{-\frac{t}{2}} & e^{-2 t} \\
\frac{1}{1+t^{2}} & \frac{1}{2+t^{2}}
\end{array}\right], \\
& A_{3}(t)=\left[\begin{array}{cc}
\frac{3}{2+t^{2}} & \frac{2}{2+t^{2}} \\
e^{-\frac{2}{3} t} & e^{-\frac{3}{4} t} t
\end{array}\right], \quad A_{4}(t)=\left[\begin{array}{cc}
e^{-3 t} & \frac{8}{1+t^{2}} \\
\frac{3}{2+t^{2}} & e^{-\frac{t}{5}}
\end{array}\right], \quad A_{5}(t)=\left[\begin{array}{cc}
e^{-\frac{2}{7} t} & e^{-\frac{3}{2} t} \\
\frac{1}{1+2 t^{2}} & \frac{1}{1+t+2 t^{2}}
\end{array}\right] \text {, }
\end{aligned}
$$

and

$$
\begin{aligned}
& B_{1}(t)=\left[\begin{array}{cc}
\frac{3}{3+t^{3}} & \frac{2}{3+t^{3}} \\
e^{-\frac{2}{3} t} & e^{-\frac{5}{4} t}
\end{array}\right], \quad B_{2}(t)=\left[\begin{array}{cc}
2 e^{-\frac{3}{2} t} & \frac{4}{1+t^{3}} \\
\frac{2}{1+t^{3}} & 2 e^{-t}
\end{array}\right] \\
& B_{3}(t)=\left[\begin{array}{cc}
3 e^{-\frac{t}{4}} & e^{-\frac{t}{2}} \\
\frac{5}{1+t^{3}} & \frac{3}{2+t^{3}}
\end{array}\right], \quad B_{4}(t)=\left[\begin{array}{cc}
\frac{2}{2+t^{3}} & \frac{1}{2+t^{3}} \\
e^{-\frac{t}{3}} & e^{-t}
\end{array}\right], \quad B_{5}(t)=\left[\begin{array}{cc}
e^{-\frac{t}{2}} & \frac{2}{2+t^{3}} \\
\frac{1}{2+t+t^{3}} & 3 e^{-\frac{t}{2}}
\end{array}\right] .
\end{aligned}
$$

The switching law of system (14) defined on the interval $[0,+\infty)$ is

$$
\Lambda=\left(\left(t_{0}, 1\right),\left(t_{1}, 5\right),\left(t_{2}, 4\right),\left(t_{3}, 2\right),\left(t_{4}, 5\right),\left(t_{5}, 3\right),\left(t_{6}, 1\right),\left(t_{7}, 2\right)\right),
$$

where the switching moments $t_{k}(k=0,1, \cdots, 7)$ are given in the following:

$$
t_{0}=0, t_{1}=7, t_{2}=12, t_{3}=20, t_{4}=29, t_{5}=46, t_{6}=60, t_{7}=94 .
$$

By employing the first equality in Equation (2), it is not difficult to obtain that

$$
\begin{aligned}
& \left\|A_{1}(t)\right\|=e^{-\frac{t}{4}}+\frac{5}{1+t^{2}}, \quad\left\|A_{2}(t)\right\|=e^{-\frac{t}{2}}+\frac{1}{1+t^{2}}, \\
& \left\|A_{3}(t)\right\|=e^{-\frac{2}{3} t}+\frac{3}{2+t^{2}}, \quad\left\|A_{4}(t)\right\|=e^{-\frac{t}{5}}+\frac{8}{1+t^{2}}, \quad\left\|A_{5}(t)\right\|=e^{-\frac{2}{7} t}+\frac{1}{1+2 t^{2}},
\end{aligned}
$$

and

$$
\begin{aligned}
& \left\|B_{1}(t)\right\|=e^{-\frac{2}{3} t}+\frac{3}{3+t^{3}}, \quad\left\|B_{2}(t)\right\|=2 e^{-t}+\frac{4}{1+t^{3}}, \\
& \left\|B_{3}(t)\right\|=3 e^{-\frac{t}{4}}+\frac{5}{1+t^{3}}, \quad\left\|B_{4}(t)\right\|=e^{-\frac{t}{3}}+\frac{2}{2+t^{3}}, \quad\left\|B_{5}(t)\right\|=3 e^{-\frac{t}{2}}+\frac{2}{2+t^{3}} .
\end{aligned}
$$

Then, with the help of the two equalities in Equation (3), we know that for $t \in[0,+\infty)$

$$
\begin{aligned}
& \|A(t)\|=\sup _{i(k)}\left\{\left\|A_{i(k)}(t)\right\| \mid i(k)=1,2, \cdots, 5\right\}=e^{-\frac{t}{5}}+\frac{8}{1+t^{2}}, \\
& \|B(t)\|=\sup _{i(k)}\left\{\left\|B_{i(k)}(t)\right\| \mid i(k)=1,2, \cdots, 5\right\}=3 e^{-\frac{t}{4}}+\frac{5}{1+t^{3}} .
\end{aligned}
$$


Through calculating, we have

$$
\begin{aligned}
\int_{0}^{+\infty}\|A(t)\| d t & =\int_{0}^{+\infty}\left(e^{-\frac{t}{5}}+\frac{8}{1+t^{2}}\right) d t=5+4 \pi<+\infty \\
\int_{0}^{+\infty}\|B(t)\| d t & =\int_{0}^{+\infty}\left(3 e^{-\frac{t}{4}}+\frac{5}{1+t^{3}}\right) d t=12+\int_{0}^{1} \frac{5}{1+t^{3}} d t+\int_{1}^{+\infty} \frac{5}{1+t^{3}} d t \\
& \leq 12+\int_{0}^{1} 5 d t+\int_{1}^{+\infty} \frac{5}{1+t^{2}} d t \leq 17+\frac{5}{4} \pi<\infty
\end{aligned}
$$

which implies that $\|A(t)\|$ and $\|B(t)\|$ are both integrable on $[0,+\infty)$, so uncertain switched system (14) is stable in measure based on Theorem 3.1.

Note that uncertain switched system (14) is consist of five uncertain sub-systems. With the help of the switching law $\Lambda$ in (15), they can be written by five uncertain differential equations as follows:

sub-system 1: $\left\{\begin{array}{c}d x_{1}(t)=\left(e^{-t} x_{1}(t)+\frac{5}{1+t^{2}} x_{2}(t)\right) d t+\left(\frac{3}{3+t^{3}} x_{1}(t)+\frac{2}{3+t^{3}} x_{2}(t)\right) d C_{t} \\ d x_{2}(t)=\left(\frac{2}{1+t^{2}} x_{1}(t)+e^{-\frac{t}{4}} x_{2}(t)\right) d t+\left(e^{-\frac{2}{3} t} x_{1}(t)+e^{-\frac{5}{4} t} x_{2}(t)\right) d C_{t} \\ t \in[0,7) \cup[60,94) \\ \left(x_{1}(0), x_{2}(0)\right)=(0.5,0.5)\end{array}\right.$ sub-system 5: $\left\{\begin{aligned} d x_{1}(t) & =\left(e^{-\frac{2}{7} t} x_{1}(t)+e^{-\frac{3}{2} t} x_{2}(t)\right) d t+\left(e^{-\frac{t}{2}} x_{1}(t)+\frac{2}{2+t^{3}} x_{2}(t)\right) d C_{t}, \\ d x_{2}(t) & =\left(\frac{1}{1+2 t^{2}} x_{1}(t)+\frac{1}{1+t+2 t^{2}} x_{2}(t)\right) d t+\left(\frac{1}{2+t+t^{3}} x_{1}(t)+3 e^{-\frac{t}{2}} x_{2}(t)\right) d C_{t}, \\ t & \in[7,12) \cup[29,46),\end{aligned}\right.$ sub-system 4: $\left\{\begin{aligned} d x_{1}(t) & =\left(e^{-3 t} x_{1}(t)+\frac{8}{1+t^{2}} x_{2}(t)\right) d t+\left(\frac{2}{2+t^{3}} x_{1}(t)+\frac{1}{2+t^{3}} x_{2}(t)\right) d C_{t} \\ d x_{2}(t) & =\left(\frac{3}{2+t^{2}} x_{1}(t)+e^{-\frac{t}{5}} x_{2}(t)\right) d t+\left(e^{-\frac{t}{3}} x_{1}(t)+e^{-t} x_{2}(t)\right) d C_{t} \\ t & \in[12,20)\end{aligned}\right.$ sub-system 2: $\left\{\begin{aligned} d x_{1}(t) & =\left(e^{-\frac{t}{2}} x_{1}(t)+e^{-2 t} x_{2}(t)\right) d t+\left(2 e^{-\frac{3}{2} t} x_{1}(t)+\frac{4}{1+t^{3}} x_{2}(t)\right) d C_{t}, \\ d x_{2}(t) & =\left(\frac{1}{1+t^{2}} x_{1}(t)+\frac{1}{2+t^{2}} x_{2}(t)\right) d t+\left(\frac{2}{1+t^{3}} x_{1}(t)+2 e^{-t} x_{2}(t)\right) d C_{t}, \\ t & \in[20,29) \cup[94,+\infty),\end{aligned}\right.$ sub-system 3: $\left\{\begin{aligned} d x_{1}(t) & =\left(\frac{3}{2+t^{2}} x_{1}(t)+\frac{2}{2+t^{2}} x_{2}(t)\right) d t+\left(e^{-\frac{2}{3} t} x_{1}(t)+e^{-\frac{3}{4} t} x_{2}(t)\right) d C_{t}, \\ d x_{2}(t) & =\left(3 e^{-\frac{t}{4}} x_{1}(t)+e^{-\frac{t}{2}} x_{2}(t)\right) d t+\left(\frac{5}{1+t^{3}} x_{1}(t)+\frac{3}{2+t^{3}} x_{2}(t)\right) d C_{t}, \\ t & \in[46,60) .\end{aligned}\right.$

On the basis of Definition 2.8, the corresponding ordinary differential equations of the above five uncertain sub-systems are listed as follows:

$$
\left\{\begin{aligned}
d x_{1}^{\alpha}(t) & =\left(e^{-t} x_{1}^{\alpha}(t)+\frac{5}{1+t^{2}} x_{2}^{\alpha}(t)\right) d t+\left|\frac{3}{3+t^{3}} x_{1}^{\alpha}(t)+\frac{2}{3+t^{3}} x_{2}^{\alpha}(t)\right| \frac{\sqrt{3}}{\pi} \ln \frac{1-\alpha}{\alpha} d t \\
d x_{2}^{\alpha}(t) & =\left(\frac{2}{1+t^{2}} x_{1}^{\alpha}(t)+e^{-\frac{t}{4}} x_{2}^{\alpha}(t)\right) d t+\left|e^{-\frac{2}{3} t} x_{1}^{\alpha}(t)+e^{-\frac{5}{4} t} x_{2}^{\alpha}(t)\right| \frac{\sqrt{3}}{\pi} \ln \frac{1-\alpha}{\alpha} d t \\
t & \in[0,7) \cup[60,94) \\
\left(x_{1}^{\alpha}(0),\right. & \left.x_{2}^{\alpha}(0)\right)=(0.5,0.5)
\end{aligned}\right.
$$




$$
\begin{aligned}
& \left\{\begin{aligned}
d x_{1}^{\alpha}(t) & =\left(e^{-\frac{2}{7} t} x_{1}^{\alpha}(t)+e^{-\frac{3}{2} t} x_{2}^{\alpha}(t)\right) d t+\left|e^{-\frac{t}{2}} x_{1}^{\alpha}(t)+\frac{2}{2+t^{3}} x_{2}^{\alpha}(t)\right| \frac{\sqrt{3}}{\pi} \ln \frac{1-\alpha}{\alpha} d t, \\
d x_{2}^{\alpha}(t) & =\left(\frac{1}{1+2 t^{2}} x_{1}^{\alpha}(t)+\frac{1}{1+t+2 t^{2}} x_{2}^{\alpha}(t)\right) d t+\left|\frac{1}{2+t+t^{3}} x_{1}^{\alpha}(t)+3 e^{-\frac{t}{2}} x_{2}^{\alpha}(t)\right| \frac{\sqrt{3}}{\pi} \ln \frac{1-\alpha}{\alpha} d t, \\
t & \in[7,12) \cup[29,46),
\end{aligned}\right. \\
& \left\{\begin{aligned}
d x_{1}^{\alpha}(t) & =\left(e^{-3 t} x_{1}^{\alpha}(t)+\frac{8}{1+t^{2}} x_{2}^{\alpha}(t)\right) d t+\left|\frac{2}{2+t^{3}} x_{1}^{\alpha}(t)+\frac{1}{2+t^{3}} x_{2}^{\alpha}(t)\right| \frac{\sqrt{3}}{\pi} \ln \frac{1-\alpha}{\alpha} d t \\
d x_{2}^{\alpha}(t) & =\left(\frac{3}{2+t^{2}} x_{1}^{\alpha}(t)+e^{-\frac{t}{5}} x_{2}^{\alpha}(t)\right) d t+\left|e^{-\frac{t}{3}} x_{1}^{\alpha}(t)+e^{-t} x_{2}^{\alpha}(t)\right| \frac{\sqrt{3}}{\pi} \ln \frac{1-\alpha}{\alpha} d t \\
t & \in[12,20)
\end{aligned}\right. \\
& \left\{\begin{aligned}
d x_{1}^{\alpha}(t) & =\left(e^{-\frac{t}{2}} x_{1}^{\alpha}(t)+e^{-2 t} x_{2}^{\alpha}(t)\right) d t+\left|2 e^{-\frac{3}{2} t} x_{1}^{\alpha}(t)+\frac{4}{1+t^{3}} x_{2}^{\alpha}(t)\right| \frac{\sqrt{3}}{\pi} \ln \frac{1-\alpha}{\alpha} d t, \\
d x_{2}^{\alpha}(t) & =\left(\frac{1}{1+t^{2}} x_{1}^{\alpha}(t)+\frac{1}{2+t^{2}} x_{2}^{\alpha}(t)\right) d t+\left|\frac{2}{1+t^{3}} x_{1}(t)+2 e^{-t} x_{2}(t)\right| \frac{\sqrt{3}}{\pi} \ln \frac{1-\alpha}{\alpha} d t, \\
t & \in[20,29) \cup[94,+\infty),
\end{aligned}\right. \\
& \left\{\begin{aligned}
d x_{1}^{\alpha}(t) & =\left(\frac{3}{2+t^{2}} x_{1}^{\alpha}(t)+\frac{2}{2+t^{2}} x_{2}^{\alpha}(t)\right) d t+\left|e^{-\frac{2}{3} t} x_{1}^{\alpha}(t)+e^{-\frac{3}{4} t} x_{2}^{\alpha}(t)\right| \frac{\sqrt{3}}{\pi} \ln \frac{1-\alpha}{\alpha} d t, \\
d x_{2}^{\alpha}(t) & =\left(3 e^{-\frac{t}{4}} x_{1}^{\alpha}(t)+e^{-\frac{t}{2}} x_{2}^{\alpha}(t)\right) d t+\left|\frac{5}{1+t^{3}} x_{1}^{\alpha}(t)+\frac{3}{2+t^{3}} x_{2}^{\alpha}(t)\right| \frac{\sqrt{3}}{\pi} \ln \frac{1-\alpha}{\alpha} d t \\
t & \in[46,60) .
\end{aligned}\right.
\end{aligned}
$$

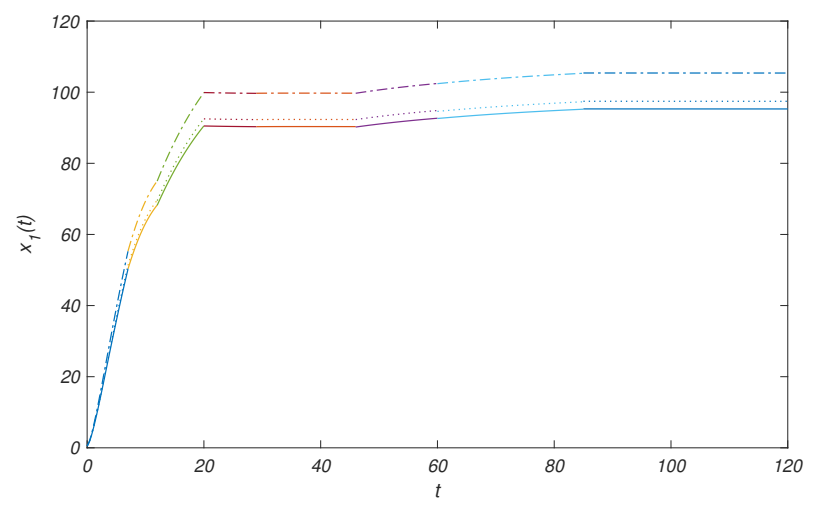

Figure 1: Trajectories of $x_{1}^{\alpha}(t)$ with initial states $(0.5,0.5),(0.51,0.51)$ and $(0.55,0.55)$.

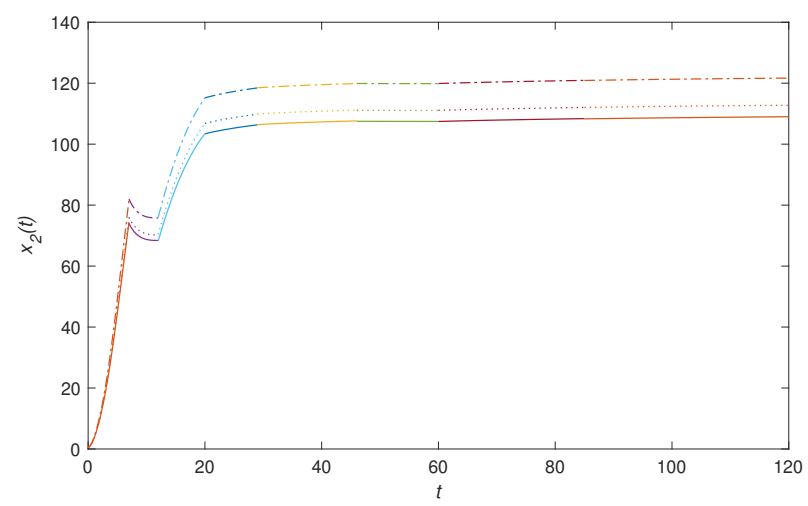

Figure 2: Trajectories of $x_{2}^{\alpha}(t)$ with initial states $(0.5,0.5),(0.51,0.51)$ and $(0.55,0.55)$. 
Figures 1 and 2 are both drawn for the above five ordinary differential equations(ODEs) when $\alpha=0.8$. In Figure 1, the solid line represents the trajectories of $x_{1}^{0.8}(t)$ with initial state $\left(x_{1}^{0.8}(0), x_{2}^{0.8}(0)\right)=(0.5,0.5)$. Two dotted lines above the solid line stand for the trajectories of $x_{1}^{0.8}(t)$ with initial states $(0.51,0.51)$ and $(0.55,0.55)$, respectively. In Figure 2 , the trajectories of $x_{2}^{0.8}(t)$ with initial states $(0.5,0.5),(0.51,0.51)$ and $(0.55,0.55)$ are illustrated by three curves from the bottom up. Observing these curves in Figures 1 and 2, when the variation of initial value becomes smaller and smaller, the corresponding solutions of these ODEs will be closer and closer as time $t$ increases. This fact indicates the stability of these five ODEs closely related to system (14), and provides us an intuitive way to comprehend stability in measure of uncertain switched system (14).

\section{Conclusions}

In this paper, stability issues of linear uncertain switched systems in infinite-time domain formulated by several uncertain differential equations were considered. On the basis of some proper premises, three types of stabilities including stability in measure, almost sure stability and stability in mean were discussed in depth, so that the internal property of linear uncertain switched systems can be illustrated clearly from different aspects. By combining stability theory and the feature of switched systems, three sufficient conditions related to the coefficient matrices were given to judge these stabilities, respectively. In addition, sufficient condition of stability in measure (or almost sure stability) is much weaker than it of stability in mean. Finally, an example was presented to intuitively display the effectiveness of the result about stability in measure.

Throughout this work, uncertain switched systems are assumed to be linear, while there exist a large number of nonlinear uncertain switched systems in practical situations. Therefore, stability and stabilization issues of nonlinear uncertain switched systems inspired by the papers [2] and [18], and optimal control subject to nonlinear switched systems with its applications on the basis of related articles[20, 21] may be investigated in the future.

\section{Acknowledgements}

This work is supported by the Natural Science Foundation of Jiangsu Province (No. BK20210633).

\section{Declarations}

Conflict of interests The authors declare that there is no conflict of interests regarding the publication of this article.

Availability of data Data sharing not applicable to this article as no datasets were generated or analyzed during the current study.

Ethics approval This article does not contain any studies with human participants performed by any of the authors.

\section{Notes on contributors}

Yadong Shu received the Ph.D. degree from Nanjing University of Science and Technology in 2018. He is a lecturer of Mathematics in Nanjing University of Information Science and Technology, Nanjing, China. His research interests include stability analysis, optimal control, switched systems and descriptor systems.

Bo Li received the Ph.D. degree from Nanjing University of Science and Technology in 2017. He is currently a lecturer with the School of Applied Mathematics, Nanjing University of Finance and Economics, Nanjing, China. His research interests are parametric optimal control and uncertain portfolio optimization. 


\section{References}

[1] Alur, R., Courcoubetis, C., Halbwachs, N.: The algorithmic analysis of hybrid systems. Theorectical Computer Science, 1995, 138(1): 3-34.

[2] Alwan, M.S., Liu, X.: Input-to-state stability for stochastic switched systems. Theory of Hybrid Systems: Deterministic and Stochastic, 117-134, Nonlinear Physical Science. Springer, Singapore, 2018.

[3] Branicky, M.S.: Multiple Lyapunov functions and other analysis tools for switched and hybrid systems. IEEE Transactions on Automatic Control, 1998, 43(4): 475-482.

[4] Bak, T., Bendtsen, J., Ravn, A.P.: Hybrid control design for a wheeled mobile robot. Hybrid Systems: Computation and Control, 2003, 2623: 50-65.

[5] Chatterjee, D., Liberzon, D.: On stability of stochastic switched systems. Proceedings of IEEE Conference on Decision and Control, 2005, 4: 4125-4127.

[6] Chen, X., Liu, B.: Existence and uniqueness theorem for uncertain differential equations. Fuzzy Optimization and Decision Making, 2010, 2(9): 69-81.

[7] Feng, W., Tian, J., Zhao, P.: Stability analysis of switched stochastic systems. Automatica, 2011, 47(1): 148-157.

[8] Filipovic, V.: Exponential stability of stochastic switched systems. Transactions of the Institute of Measurement and Control, 2009, 31(2): 205-212.

[9] Hespanha, J.P., Morse, A.S.: Stability of switched systems with average dwell-time. Proceedings of the 38th IEEE Conference on Decision and Control, 1999, 3: 2655-2660.

[10] Liberzon, D., Morse, A.S.: Basic problem in stability and design of switched systems. IEEE Control Systems Magazine, 2001, 19: 59-70.

[11] Lin, H., Antsaklis, P.J.: Stability and stabilizability of switched linear systems: a survey of recent results. IEEE Transactions on Automatic Control, 2009, 54(2): 308-322.

[12] Liu, B.: Uncertainty Theory, 2nd ed. Springer-Verlag, Berlin, Germany, 2007.

[13] Liu, B.: Fuzzy process, hybrid process and uncertain process. Journal of Uncertain Systems, 2008, 2(1): 3-16.

[14] Liu, B.: Some research problems in uncertainty theory. Journal of Uncertain Systems, 2009, 3(1): 3-10.

[15] Liu, B.: Uncertainty Theory: A Branch of Mathematics for Modeling Human Uncertainty. Springer-Verlag, Berlin, Germany, 2010.

[16] Liu, H., Ke, H., Fei, W.: Almost sure stability for uncertain differential equation. Fuzzy Optimization and Decision Making, 2014, 13(4): 463-473.

[17] Liu, J., Zhang, Y.: The stability analysis for uncertain heat equations based on $p$-th moment. Soft Computing, 2020, 24: 2833-2839.

[18] Ning, Z., Zhang, L., Lam, J.: Stability and stabilization of a class of stochastic switching systems with lower bound of sojourn time. Automatica, 2018, 92: 18-28.

[19] Phat, V.N., Sau, N.H.: On exponential stability of linear singular positive delayed systems. Applied Mathematics Letters, 2014, 38: 67-72.

[20] Sheng, L., Zhu, Y., Wang, K.: Uncertain dynamical system-based decision making with application to productioninventory problems. Applied Mathematics Modelling, 2018, 56: 275-288.

[21] Shtessel, Y.B., Raznopolov, O.A., Ozerov, L.A.: Control of multiple modular dc-to-dc power converts in conventional and dynamic sliding surfaces. IEEE Transactions on Circuits and Systems, 1998, 45(10): 1091-1100.

[22] Shu, Y., Zhu, Y.: Stability and optimal control for uncertain continuous-time singular systems. European Journal of Control, 2017, 34: 16-23.

[23] Su, T., Wu, H., Zhou, J.: Stability of mutli-dimensional uncertain differential equation. Soft Computing, 2016, 20(12): 4991-4998.

[24] Tao, N., Zhu, Y.: Stability and attractivity in optimistic value for dynamical systems with uncertainty. International Journal of General Systems, 2016, 45(4): 418-433.

[25] Yang, X., Gao, J.: Linear-quadratic uncertain differential game with application to resource extraction problem. IEEE Transactions on Fuzzy Systems, 2016, 24(4): 819-826.

[26] Yao, K.: Multi-dimensional uncertain calculus with Liu process. Journal of Uncertain Systems, 2014, 8(4): 244254. 
[27] Yao, K., Chen, X.: A numerical method for solving uncertain differential equations. Journal of Intelligent 6 Fuzzy Systems, 2013, 25(3): 825-832.

[28] Yao, K., Gao, J., Gao, Y.: Some stability theorems of uncertain differential equation. Fuzzy Optimization and Decision Making, 2013, 12(1): 3-13.

[29] Yao, K., Ke, H., Sheng, Y.: Stability in mean for uncertain differential equation. Fuzzy Optimization and Decision Making, 2015, 14(3): 365-379.

[30] Zhu, Y.: Uncertain optimal control with application to a portfolio selection model. Cybernetics and Systems, 2010, 41(7): 535-547. 